\title{
Relationship Between Clinical Variables and Health-Related Quality of Life in Young Adult Subjects With Cystic Fibrosis
}

\author{
Gabriele C Forte, Gabriela C Barni MSc, Christiano Perin PhD, Fernanda C Casarotto MD, \\ Simone C Fagondes PhD, and Paulo de Tarso Roth Dalcin PhD
}

\begin{abstract}
BACKGROUND: Health-related quality of life (HRQOL) has received much attention in patients with cystic fibrosis (CF). The goal of this study was to evaluate the association between clinical, lung function, sleep quality, and polysomnographic variables with 2 HRQOL questionnaires, the shorterversion World Health Organization Quality of Life (WHOQOL-BREF) and Cystic Fibrosis Quality of Life (CFQOL) questionnaires, in adult subjects with CF. METHODS: In a cross-sectional study, 51 subjects underwent clinical evaluation and overnight polysomnography and answered WHOQOL-BREF, CFQOL, Pittsburgh Sleep Quality Index, and Epworth Sleepiness Scale questionnaires. In addition, pulmonary function tests, 6-min walk tests, and echocardiography were performed. RESULTS: For WHOQOL-BREF scores, the sleep quality index was associated with the physical domain; the percent-of-predicted 6-min walk distance (6MWD) and sleepiness scale were associated with the psychological domain; the percent-of-predicted $\mathrm{FEV}_{1}$ and sleep quality index were associated with the social relationship domain; and the sleep quality index was associated with the environment domain. For CFQOL scores, age at diagnosis, clinical score, and sleep quality index were associated with the physical functioning domain; the percent-of-predicted 6MWD and pulmonary arterial systolic pressure were associated with the role domain; sex and sleep quality index were associated with the vitality domain; the apnea-hypopnea index was associated with the emotional functioning domain; sex and body mass index (BMI) were associated with the body image domain; the percent-of-predicted 6MWD and sleep quality index were associated with the health perception domain; age, sex, BMI, and arousal index were associated with the weight domain; age, sex, percent-of-predicted $\mathrm{FEV}_{1}$, percent-of-predicted $6 \mathrm{MWD}$, and pulmonary arterial systolic pressure were associated with the respiratory symptom domain; and the clinical score was associated with the digestive symptom domain. CONCLUSIONS: The sleep quality index score, 6MWD, sleepiness scale score, and $\mathrm{FEV}_{1}$ were predictors of WHOQOL-BREF scores. Age at diagnosis, clinical score, sleep quality score, 6MWD, sex, apnea-hypopnea index, BMI, current age, arousal index, $\mathbf{F E V}_{1}$, and pulmonary arterial systolic pressure were predictors of CFQOL scores. Key words: cystic fibrosis; quality of life; sleep disorders; respiratory function tests; 6-min walk test. [Respir Care 2015;60(10):1459-1468. ( ) 2015 Daedalus Enterprises]
\end{abstract}

\section{Introduction}

Cystic fibrosis (CF) is a progressive genetic disease that affects mainly the white population. The median predicted life expectancy for patients with CF has risen steadily in the last years. In 2012, the median predicted survival age was $41.1 \mathrm{y}$, with $>49 \%$ adults with CF reaching this age. ${ }^{1}$ However, standard treatments are rigorous, requiring time-consuming regimens

\footnotetext{
Ms Forte, Ms Barni, and Drs Perin, Fagondes, and Dalcin are affiliated with the Universidade Federal do Rio Grande do Sul (UFRGS), Porto Alegre, Rio Grande do Sul, Brazil. Dr Casarotto is affiliated with the Hospital de Clínicas de Porto Alegre, Universidade Federal do Rio Grande do Sul, Porto Alegre, Rio Grande do Sul, Brazil. Drs Fagondes and Dalcin are also affiliated the Pneumology Department, Hospital de Clínicas de Porto Alegre, Porto Alegre, Rio Grande do Sul, Brazil.
} This work was supported by the Fundo de Pesquisa do Hospital de
Clínicas de Porto Alegre (FIPE-HCPA) and the Coordenação de Aper-
feiçoamento de Pessoal de Nível Superior/Conselho Nacional de Desen-
volvimento Científico e Tecnológico (CAPES/CNPq). Dr Casarotto re-
ceived a scientific initiation scholarship from UFRGS-CNPq. Ms Forte
and Ms Barni received fellowships from CAPES/CNPq. The authors
have disclosed no conflicts of interest. 
of patient self-care. Daily management routinely involves airway clearance therapies, exercise, oral and inhaled respiratory medications, and oral supplementation with pancreatic enzymes and vitamins. ${ }^{2}$

Because life expectancy has improved markedly, healthrelated quality of life (HRQOL) research has gained substantial interest in this population. HRQOL is a multidimensional construct that encompasses physical, psychological, social, and functional domains of daily life and measures the impact of health and illness on these areas of functioning. ${ }^{3} \mathrm{HRQOL}$ measurement has been used in clinical trials,,${ }^{4,5}$ and reviews $s^{6-8}$ have reported on HRQOL as an outcome measurement in clinical trials in adults. HRQOL measurement can provide a standardized, valid, and reliable way to obtain a subject's perspective as to the benefits and limitation of a specific intervention. ${ }^{3}$

The World Health Organization Quality of Life (WHOQOL) Group developed an international cross-culturally comparable quality-of-life assessment instrument. ${ }^{9}$ Its shorter version (WHOQOL-BREF) ${ }^{10}$ comprises 26 items of the original instrument, which aimed to encompass the general domains of HRQOL: physical health, psychological health, social relationships, and environment. It has been used in several countries and in different populations, showing many possibilities for use in international and multicultural perspectives. Moreover, a disease-specific HRQOL questionnaire has been developed for patients with $\mathrm{CF}$. The Cystic Fibrosis Quality of Life (CFQOL) questionnaire ${ }^{11,12}$ is a validated disease-specific HRQOL measure of both physical and psychosocial aspects of health. Physical HRQOL domains include physical functioning, body image, eating disturbances, digestive symptoms, respiratory symptoms, weight, health perceptions, and vitality, whereas psychosocial domains include treatment burden and emotional, social, and role functioning.

The literature shows various factors that are associated with quality-of-life domains in subjects with CF.13-15 Although several studies have looked at HRQOL in subjects with $\mathrm{CF}$, few have investigated the impact of sleep quality and sleep-disordered breathing in daily life. ${ }^{16}$ Nocturnal oxygen desaturation was associated with impaired HRQOL independent of the effects of lung function and awake gas exchange in stable out-patients with $\mathrm{CF}$ and moderate-tosevere lung disease. In addition, it is important to evaluate the HRQOL in different populations of adults with $\mathrm{CF}$

\footnotetext{
Correspondence: Paulo de Tarso Roth Dalcin PhD, Departamento de Medicina Interna, Faculdade de Medicina, Universidade Federal do Rio Grande do Sul, Rua Honório Silveira Dias 1529/901, Bairro São João, Porto Alegre, Rio Grande do Sul 90.540-070, Brazil. E-mail: pdalcin@terra.com.br.
}

DOI: $10.4187 /$ respcare. 03665

\section{QUICK LOOK}

\section{Current knowledge}

Cystic fibrosis (CF) is a progressive genetic disease affecting mainly the white population and is associated with a median survival age of $41 \mathrm{y}$. Daily disease management involves airway clearance therapies, exercise, oral and inhaled respiratory medications, and dietary supplements with pancreatic enzymes and vitamins. Factors associated with quality-of-life domains in patients with CF include physical functioning, body image, eating disturbances, digestive symptoms, respiratory symptoms, weight, health perceptions, and vitality, as well as psychosocial domains, including treatment burden, emotional, social, and role functioning.

\section{What this paper contributes to our knowledge}

In adult subjects with CF, 2 health-related quality of life (HRQOL) questionnaires were compared: the shorterversion World Health Organization Quality of Life (WHOQOL-BREF) and the Cystic Fibrosis Quality of Life (CFQOL) questionnaires. Sleep quality index score, 6-min walk distance (6MWD), sleepiness scale score, and $\mathrm{FEV}_{1}$ were predictors of WHOQOL-BREF scores. Age at diagnosis, clinical score, sleep quality score, 6MWD, sex, apnea-hypopnea index, body mass index, current age, arousal index, and $\mathrm{FEV}_{1}$, were predictors of CFQOL scores. These findings highlight the important role of different HRQOL measures in specific subjective health perception in subjects with CF. HRQOL together with pulmonary function, 6MWD, and sleep/ sleepiness scale scores provide a global assessment of subjects with $\mathrm{CF}$.

with regard to clinical, nutritional, pulmonary function, and sleep quality aspects, as well as to report international and multicultural differences. The aim of this study was to evaluate the association between clinical, nutritional, lung function, sleep quality, and polysomnographic variables with 2 quality-of-life instruments, the WHOQOL-BREF and CFQOL questionnaires, in adult subjects with $\mathrm{CF}$ at a large tertiary care center in southern Brazil.

\section{Methods}

\section{Subjects}

This cross-sectional study recruited subjects regularly attending the adult CF out-patient clinic at the Hospital de Clínicas de Porto Alegre in Rio Grande do Sul, Brazil. Subjects were eligible if they had a confirmed diagnosis 
either by at least 2 abnormal sweat chloride tests and/or by a genetic test according to international guidelines ${ }^{17}$ and if they were $\geq 16$ y of age. Exclusion criteria were: pulmonary exacerbation in the previous $30 \mathrm{~d}$, cardiac or neurological disease or other chronic disease that limited the study procedures, current treatment with sedative or antiepileptic drugs, and pregnancy. The study was approved by the human research ethics committee, and informed consent was obtained from adult subjects and from the parents of subjects $<18$ y old.

During the study period, 67 subjects attended the adult CF clinic at the Hospital de Clínicas de Porto Alegre. Seven subjects did not have a diagnosis of CF confirmed by sweat chloride test or genetic test. Six subjects declined to participate in the study, and 3 subjects did not undergo all study evaluations. Therefore, 51 subjects were included in the study.

\section{Procedures}

Subjects underwent a comprehensive clinical and nutritional evaluation and answered questionnaires about sleep quality and HRQOL during an out-patient clinic visit. On the following day, forced spirometry, chest radiograph, Doppler echocardiography, 6-min walk test, and overnight polysomnography were performed in this order for each subject. The researchers who performed the daytime investigations were blinded to the subjects' clinical and functional status. Demographic data (current age, sex, and age and time of diagnosis) were obtained from the subjects' medical records on the date that the questionnaires were completed.

\section{HRQOL}

HRQOL was measured using the WHOQOL-BREF 18 and $\mathrm{CFQOL}^{19}$ questionnaires, which are Portuguese validated. The CFQOL questionnaire contains 44 questions and consists of the following domains: physical functioning, role, vitality, emotional functioning, social, body image, eating disturbances, treatment burden, health perceptions, weight, respiratory symptoms, and digestive symptoms. Response choices generally included ratings of frequency and difficulty on a 4-point scale (1 [always] to 4 [never], $1=$ a lot of difficulty to $4=$ no difficulty) or true/false responses $(1=$ very true to $4=$ very false $)$. Scores were calculated from 0 to 100 points, where higher scores in each domain represented better HRQOL. The WHOQOL-BREF is a short questionnaire of 24 items across 4 scales: physical, psychological, social relationships, and environment. Scores range from 0 to 100 , with higher scores indicating better HRQOL and functioning.

\section{Pulmonary Function Test}

Pulmonary function was determined according to the spirometry protocol following the American Thoracic Society criteria. ${ }^{20} \mathrm{FVC}$ and pre- and post-bronchodilator $\mathrm{FEV}_{1}$ (L and percent-of-predicted) for age, height, and sex were calculated. ${ }^{21}$

\section{Clinical and Radiographic Scores}

Clinical scores were obtained using the ShwachmanKulczycki scoring system. ${ }^{22}$ A conventional chest radiograph was obtained, and a pulmonologist blinded to subject clinical status determined the radiographic scores according to the scoring system of Brasfield et al. ${ }^{23}$

\section{Echocardiography}

Doppler echocardiography (ATL-HDI 5000, Advanced Technology Laboratories, Bothell, Washington) was performed by a board-certified senior echocardiographer according to international recommendations. ${ }^{24}$ Pulmonary artery systolic pressure was obtained from the estimated tricuspid regurgitation gradient and the estimated right atrial pressure.

\section{6-min Walk Test}

The 6-min walk test was performed following a standardized protocol. ${ }^{25}$ The total distance walked and the initial and final $\mathrm{S}_{\mathrm{pO}_{2}}$ were recorded. The 6-min walk distance (6MWD) was expressed as percent of predicted using a reference equation for the Brazilian population. ${ }^{26}$

\section{Sleep Questionnaires}

The Pittsburgh Sleep Quality Index (PSQI) ${ }^{27}$ and Epworth Sleepiness Scale (ESS) ${ }^{28}$ questionnaires were answered by subjects on the night of the diagnostic sleep study. The PSQI is a self-administered questionnaire that assesses sleep quality and disturbance. Seven components are combined to produce a global score ranging from 0 to 21. A score of $>5$ indicates sensitive and specific measures of poor sleep quality. The ESS is a validated sleep and self-administered questionnaire in which a score of $>10$ indicates subjective daytime sleepiness. Both questionnaires are validated and adapted to the Brazilian Portuguese language. 29,30

\section{Polysomnography}

Standard overnight sleep studies were performed using a computerized system (Neurofax EEG-9100, Nihon Kohden, Tokyo, Japan). Awake $\mathrm{S}_{\mathrm{pO}_{2}}$ was estimated by calcu- 
lating the mean of these values at rest, in a sitting position, and during a 5-min period of wakefulness at the beginning of the study. Sleep staging, arousal analysis, and scoring of respiratory events during sleep were performed according to the American Academy of Sleep Medicine. ${ }^{31}$ Significant sleep desaturation was defined as an $\mathrm{S}_{\mathrm{pO}_{2}}$ during sleep of $<90 \%$ for $\geq 5$ min with a nadir of at least $85 \% .32$

\section{Statistical Analysis}

Descriptive statistics (mean $\pm \mathrm{SD}$, range, $n$, and proportion) were calculated for characteristics of the study sample (Table 1). A separate regression model was constructed for each of the 12 CFQOL domains and 4 WHOQOLBREF domains. Hierarchical logistic regression models with predictors added one at a time were also examined to evaluate the possible collinearity among the predictors. The predictors selected in the final model were based on both numerical and clinical importance. We first constructed regression models for each of the CFQOL and WHOQOL-BREF domains using demographic, anthropometric, and clinical data. Univariate analysis was then performed using simple linear correlations to explore the relationships between demographic, clinic, and anthropometric data and HRQOL measures (WHOQOL-BREF and CFQOL). Where a significant relationship was identified $(P<.10)$, a stepwise multiple regression was performed using the CFQOL and WHOQOL-BREF scores as dependent variables and significant associated demographic, clinical, and anthropometric characteristics as independent variables. Regression analysis was performed using a level of significance of $P<.05$. All analyses were performed using SPSS 18.0 (IBM, Armonk, New York).

We calculated the sample size based on the study of Quittner et $\mathrm{al}^{11}$ using the physical functioning CFQOL dimension and percent-of-predicted $\mathrm{FEV}_{1}$. Considering $r=0.42$ with a power of $80 \%$ and a level of significance of $.05,47$ subjects would be needed in the study.

\section{Results}

Fifty-one clinically stable subjects with CF participated in the study; $47 \%$ were female. The mean age was $25.1 \pm 8.8 \mathrm{y}$, the median age at diagnosis was $3 \mathrm{y}$, and the mean body mass index (BMI) was $20.5 \mathrm{~kg} / \mathrm{m}^{2}$. Additional clinical data and other characteristics are provided in Table 1 .

Tables 2 and 3 provide descriptive statistics for the WHOQOL-BREF and CFQOL scores, respectively. The highest WHOQOL-BREF scores were obtained for the social relationship domain $(71 \pm 21)$, and the lowest for the physical health domain $(63 \pm 18)$. The highest CFQOL scores were obtained for the eating disturbance $(85 \pm 17)$ and digestive symptom $(83 \pm 15)$ domains. The lowest CFQOL
Table 1. Demographics and Clinical Characteristics of Adolescent and Adult Subjects With Cystic Fibrosis

\begin{tabular}{|c|c|}
\hline Variable & Values \\
\hline \multicolumn{2}{|l|}{$\operatorname{Sex}, n(\%)$} \\
\hline Female & $24(47.1)$ \\
\hline Male & $27(52.9)$ \\
\hline Age, mean $\pm \mathrm{SD}$ y & $25.1 \pm 8.8$ \\
\hline Age at diagnosis, median y (IQR) & $3(21)$ \\
\hline Time of diagnosis, median y (IQR) & $15.6(16)$ \\
\hline $\mathrm{BMI}$, mean $\pm \mathrm{SD} \mathrm{kg} / \mathrm{m}^{2}$ & $20.5 \pm 2.4$ \\
\hline Shwachman-Kulczycki score, median (IQR) & $70(15)$ \\
\hline $\begin{array}{l}\text { Pulmonary artery systolic pressure, } \\
\text { mean } \pm \mathrm{SD} \mathrm{mm} \mathrm{Hg}\end{array}$ & $29.7 \pm 7.9$ \\
\hline ESS score, mean $\pm \mathrm{SD}$ & $8.2 \pm 3.5$ \\
\hline $\mathrm{PSQI}$ score, mean $\pm \mathrm{SD}$ & $6.0 \pm 3.5$ \\
\hline Arousal index score, mean $\pm \mathrm{SD}$ & $12.1 \pm 6.1$ \\
\hline \multicolumn{2}{|l|}{ Sleep stage score, mean $\pm \mathrm{SD}$} \\
\hline Stage $1 / 2$ sleep & $62.1 \pm 11.8$ \\
\hline Stage 3 sleep & $20.0 \pm 7.1$ \\
\hline Stage REM sleep & $18.3 \pm 6.2$ \\
\hline AHI, median events/h (IQR) & $0.3(0.55)$ \\
\hline \multicolumn{2}{|l|}{$\mathrm{S}_{\mathrm{pO}_{2}}$, mean $\pm \mathrm{SD} \%$} \\
\hline Awake & $95.1 \pm 2.5$ \\
\hline Mean sleep & $92.0 \pm 3.5$ \\
\hline Minimum sleep & $85.8 \pm 6.3$ \\
\hline \multicolumn{2}{|l|}{$\mathrm{S}_{\mathrm{PO}_{2}}<90 \%$ of predicted, mean $\pm \mathrm{SD}$} \\
\hline$\%$ of total sleep time & $22.8 \pm 37.7$ \\
\hline$\%$ of REM sleep & $23.7 \pm 39.2$ \\
\hline Brasfield score, median (IQR) & $14(7)$ \\
\hline $\mathrm{FEV}_{1}$, mean $\pm \mathrm{SD} \%$ predicted & $57.7 \pm 24.7$ \\
\hline $\mathrm{FVC}$, mean $\pm \mathrm{SD} \%$ predicted & $69.8 \pm 23.2$ \\
\hline $6 \mathrm{MWD}$, mean $\pm \mathrm{SD} \mathrm{m}$ & $544.2 \pm 133.7$ \\
\hline $6 \mathrm{MWD}$, mean $\pm \mathrm{SD} \%$ predicted & $84.9 \pm 20.0$ \\
\hline $\mathrm{S}_{\mathrm{pO}_{2}}$ at rest during $6 \mathrm{MWT}$, mean $\pm \mathrm{SD} \%$ & $96.3 \pm 2.3$ \\
\hline $\mathrm{S}_{\mathrm{pO}_{2}}$ at end of $6 \mathrm{MWT}$, mean $\pm \mathrm{SD} \%$ & $93 \pm 7.6$ \\
\hline $\begin{array}{l}N=51 \\
\mathrm{IQR}=\text { interquartile range } \\
\text { BMI }=\text { body mass index } \\
\text { ESS }=\text { Epworth Sleepiness Scale } \\
\text { PSQI = Pittsburgh Sleep Quality Index } \\
\text { REM = rapid eye movement } \\
\text { AHI = apnea-hypopnea index } \\
6 \mathrm{MWD}=6 \text {-min-walk distance } \\
6 \mathrm{MWT}=6 \text {-min-walk test }\end{array}$ & \\
\hline
\end{tabular}

scores were obtained for the health perception domain $(53 \pm 28)$.

To examine the relationship between clinical variables and HRQOL as indicated by the WHOQOL-BREF and CFQOL scores, we constructed multivariable linear regression models controlling for age and sex. The results of the simple and multiple linear regression analyses are shown in Tables 4 and 5.

In multiple regression models for the 4 WHOQOL-BREF domains, the PSQI score associated with a less favorable physical domain; the percent-of-predicted 6MWD (positively) and ESS score (negatively) were associated with 
Table 2. WHOQOL-BREF Scores

\begin{tabular}{lc}
\hline \hline \multicolumn{1}{c}{ Domain } & WHOQOL-BREF Scores \\
\hline Physical health & $63 \pm 18$ \\
Psychological health & $70 \pm 15$ \\
Social relationship & $71 \pm 21$ \\
Environment & $67 \pm 13$ \\
& \\
Values are presented as mean \pm SD. & \\
WHOQOL-BREF $=$ shorter-version World Health Organization Quality of Life \\
\hline
\end{tabular}

Table 3. CFQOL Scores

\begin{tabular}{lc}
\hline \hline \multicolumn{1}{c}{ Domain } & CFQOL Scores \\
\hline Physical functioning & $55 \pm 32$ \\
Role & $74 \pm 22$ \\
Vitality & $61 \pm 22$ \\
Emotional functioning & $73 \pm 18$ \\
Social functioning & $62 \pm 17$ \\
Body image & $76 \pm 22$ \\
Eating disturbances & $85 \pm 17$ \\
Treatment burden & $59 \pm 22$ \\
Health perceptions & $53 \pm 28$ \\
Weight & $57 \pm 37$ \\
Respiratory symptoms & $60 \pm 17$ \\
Digestive symptoms & $83 \pm 15$ \\
\hline Values are presented as mean \pm SD. & \\
CFQOL $=$ Cystic Fibrosis Quality of Life & \\
\hline
\end{tabular}

the psychological domain; the percent-of-predicted $\mathrm{FEV}_{1}$ (positively) and PSQI score (negatively) were associated with the social relationship domain; and the PSQI score was inversely associated with the environment domain. In multiple regression models for the CFQOL domains, age at diagnosis (negatively), Shwachman-Kulczycki score (positively), and PSQI score (negatively) were associated with the physical functioning domain; the percent-of-predicted 6MWD was positively associated with the role domain; male sex and PSQI score were negatively associated with the vitality domain; the apnea-hypopnea index was negatively associated with the emotional functioning domain; male sex and BMI were positively associated with the body image domain; the percent-of-predicted 6MWD was positively associated with the health perception domain; age (negatively), male sex (positively), BMI (positively), and arousal index (negatively) were associated with the weight domain; age (negatively), sex (positively), percent-of-predicted $\mathrm{FEV}_{1}$ (positively), percent-of-predicted 6MWD (positively), and pulmonary artery systolic pressure (negatively) were associated with the respiratory symptom domain; and the Shwachman-Kulczycki score were negatively associated with the digestive symptom domain.

\section{Discussion}

This cross-sectional study evaluated HRQOL in clinically stable young adult subjects with $\mathrm{CF}$ at a large tertiary care center in southern Brazil using a generic questionnaire (WHOQOL-BREF) and a specific questionnaire (CFQOL). The lowest WHOQOL-BREF score was obtained for the physical health domain, and the lowest CFQOL score was obtained for the health perception domain. The PSQI score, percent-of-predicted 6MWD, ESS score, and percent-of-predicted $\mathrm{FEV}_{1}$ were independent predictors of WHOQOL-BREF scores. Age at diagnosis, Shwachman-Kulczycki score, PSQI score, percent-of-predicted 6MWD, pulmonary artery systolic pressure, sex, apnea-hypopnea index, BMI, current age, arousal index, percent-of-predicted $\mathrm{FEV}_{1}$, and were independent predictors of CFQOL scores.

Measures of HRQOL are also important for identifying the benefits of new treatments that are demonstrated in conventional health indexes, such as pulmonary function testing. Multiple HRQOL instruments have been used previously to describe subject-related outcomes. ${ }^{11,12,33,34}$ In 1996, the first CF-specific quality-of-life instrument (Cystic Fibrosis Questionnaire [CFQ]) was developed, ${ }^{35}$ and in 2006, the United States Cystic Fibrosis Foundation developed an English translation of the CFQ. ${ }^{11}$ Increases in life expectancy for patients with CF have a strong impact on measuring HRQOL using a disease-specific instrument and a general quality-of-life instrument such as the WHOQOLBREF questionnaire.

We obtained higher PSQI scores in this study, suggesting poor sleep quality. These higher scores were correlated with lower scores in CFQOL domains, such as physical functioning, vitality, and health perception. Regarding the WHOQOL-BREF questionnaire, we observed a correlation between 3 domains (physical, social relationships, and environment). However, Bouka et $\mathrm{al}^{36}$ observed that higher PSQI scores correlated with lower scores in the vitality, emotional functioning, social, and role domains. Still, in relation to sleep quality, statistical analysis showed that daytime sleepiness, as evaluated by the ESS, was significantly related to the psychological domain in the WHOQOLBREF questionnaire. The ESS scores did not significantly correlate with the CFQOL domains. The arousal index correlated only with the CFQOL weight domain.

The young adult population in this study presented with significant disease severity with low clinical scores, moderate impairment of nutritional status, poor pulmonary function, and mild reduction in exercise capacity. At baseline, we found that lung function and nutritional status were similar to those in prior studies..$^{11,36-38}$ These factors surely have had a great impact on HRQOL outcomes.

We found an association between the percent-of-predicted $\mathrm{FEV}_{1}$ and WHOQOL-BREF and CFQOL scores. 


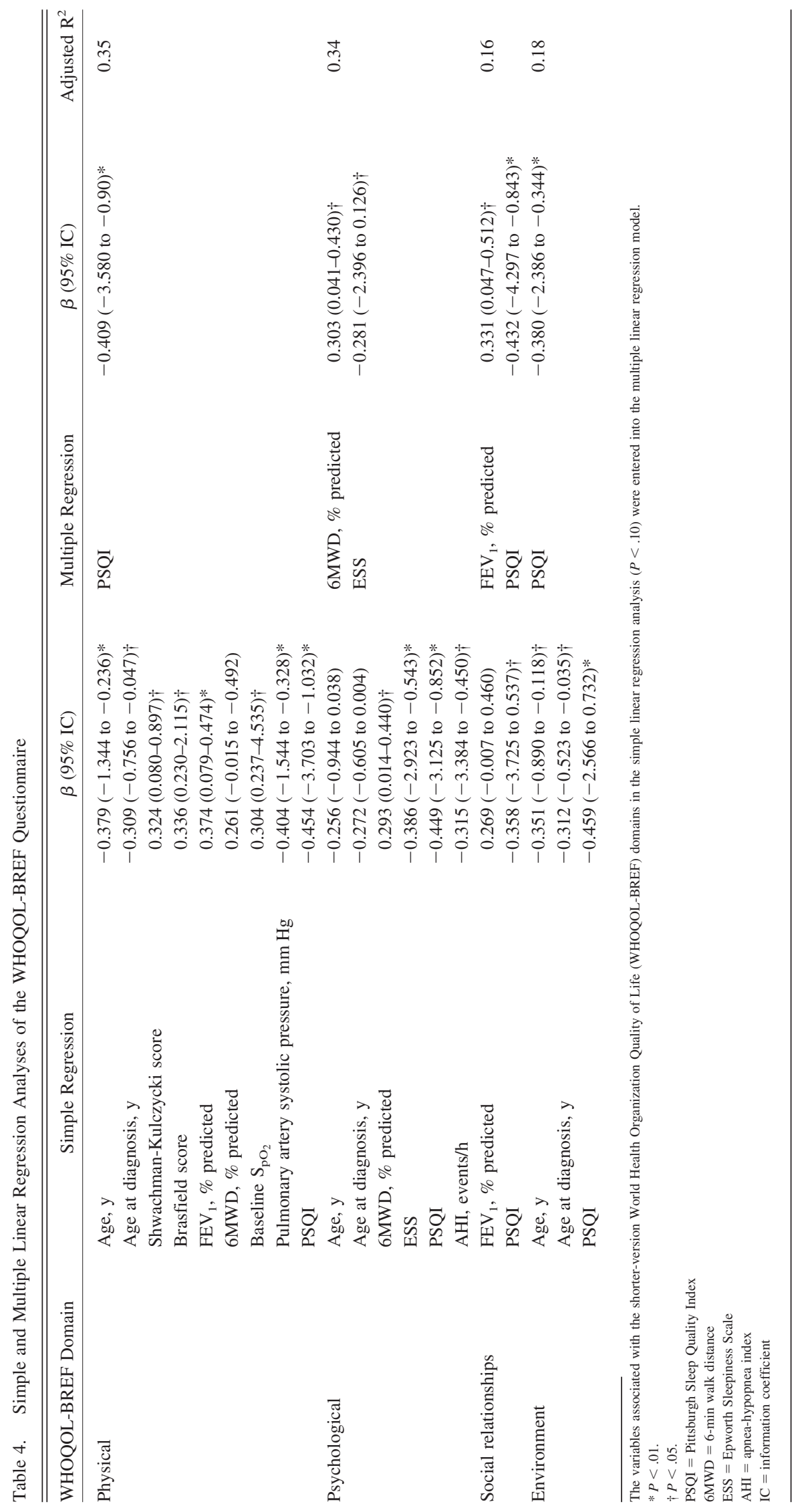


HRQOL IN CF

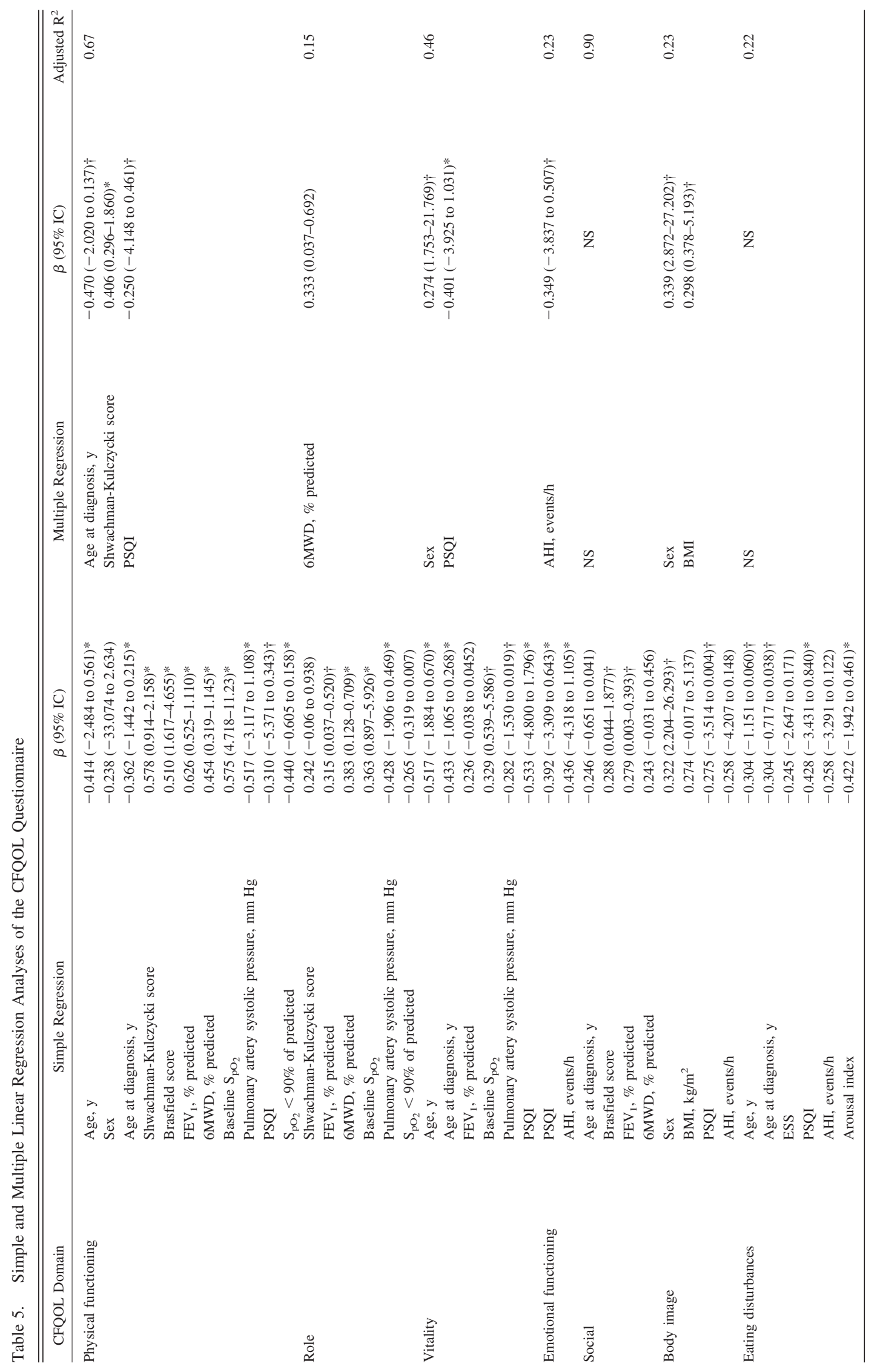




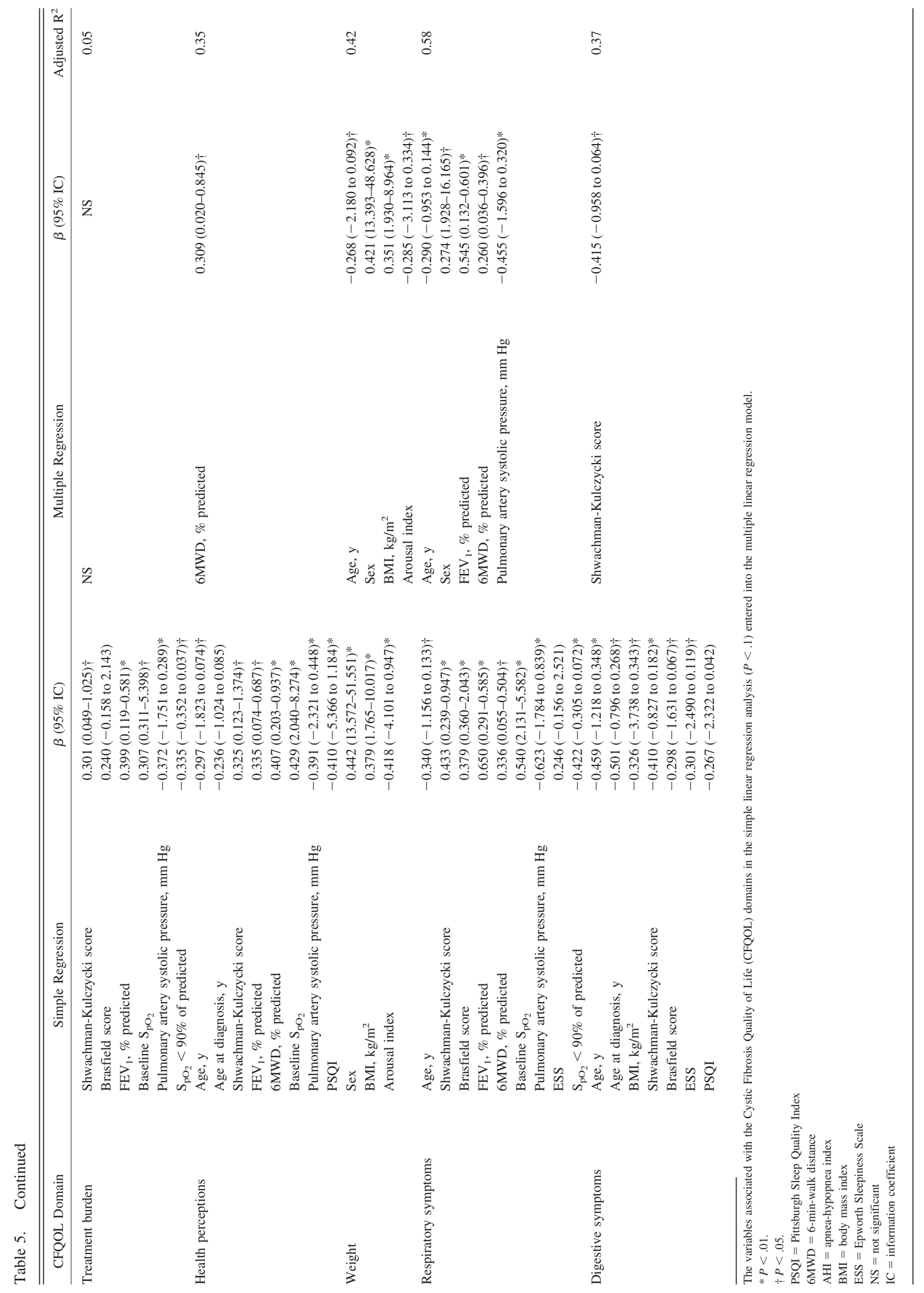


Previous studies showed that pulmonary function in adults with $\mathrm{CF}$ is also a significant predictor of CFQOL scores. ${ }^{38,39}$ In a longitudinal study, Abbott et $\mathrm{al}^{38}$ observed that the percent-of-predicted $\mathrm{FEV}_{1}$ significantly correlated with all domains except body image. In contrast, in the present study, the percent-of-predicted $\mathrm{FEV}_{1}$ showed an association only with the social relationship domain in the WHOQOLBREF questionnaire and the respiratory symptom domain in the CFQOL questionnaire. When specific instruments are used, this correlation becomes more consistent. Measurement with different HRQOL instruments provides additional information on the impact of this disease that cannot be obtained by physiological tests such as pulmonary function testing.

BMI and percent-of-predicted $\mathrm{FEV}_{1}$ are important indicators of disease status in patients with $\mathrm{CF}$, and those patients with better nutritional status report a better HRQOL.${ }^{40}$ In the present study, there was no evidence of association between BMI and any of the WHOQOL-BREF domain scores, although BMI was a predictor of CFQOL scores. Similar results were demonstrated by Dill et al ${ }^{41}$ in a longitudinal study with subject-reported outcomes: nutritional status was associated with CFQ-Revised physical domain scores. Our study demonstrated a significant effect for sex as a predictor of CFQOL scores for the weight and body image domains, but there were no associations with any WHOQOL-BREF scores. In a previous study, female sex was associated with higher scores in body image and weight scales, and females reported lower physical functioning and vitality scores. ${ }^{39}$ However, Shoff et al ${ }^{40}$ found no significant sex differences in mean CFQ dimension scores or nutritional status scores in children and adolescents, but the strength of association between nutritional status and CFQ scores differed between boys and girls for some dimensions, such as physical functioning. In the present study, the 6MWD was an independent predictor for both WHOQOL-BREF and CFQOL scores. Bradley et al ${ }^{42}$ demonstrated that the performance in the modified shuttle test was significantly associated with the change in quality of life according to the Chronic Respiratory Disease Questionnaire. ${ }^{43}$ The 6-min walk test is a useful measure of functional capacity, and it has been widely used to measure the response to therapeutic interventions for pulmonary disease such as $\mathrm{CF}^{4}{ }^{4}$

This study has some potential limitations that should be addressed. It is a cross-sectional study, and therefore, it is not possible to establish the temporal sequence between the factors studied and HRQOL. In addition, this investigation was done in a single center with a small sample size. Notwithstanding, the study was performed in the largest CF center in southern Brazil, and the factors identified as predictors of HRQOL would be relevant for clinical care and clinical research in CF.
Although there are several studies on the subject, our findings contribute to raising the awareness of the multifactorial aspects, including polysomnographic variables, related to HRQOL in subjects with $\mathrm{CF}$, and we also evaluated 2 quality-of-life instruments (WHOQOL-BREF and CFQOL). In the attempt to improve HRQOL in clinical practice, the $\mathrm{CF}$ multidisciplinary team should take into account all relevant clinical, nutritional, pulmonary function, and sleep quality data under evaluation.

\section{Conclusions}

In summary, in adult subjects with CF, PSQI score, 6MWD, ESS score, and percent-of-predicted $\mathrm{FEV}_{1}$ were independent predictors of WHOQOL-BREF scores. Age at diagnosis, Shwachman-Kulczycki score, PSQI score, 6MWD, sex, apnea-hypopnea index, BMI, current age, arousal index, percent-of-predicted $\mathrm{FEV}_{1}$, and pulmonary artery systolic pressure were independent predictors of CFQOL scores. Therefore, the findings support the important role of different quality-of-life measures in specific subjective health perception in subjects with CF. Healthcare professionals should consider routinely evaluating HRQOL together with pulmonary function, 6MWD, and sleep/sleepiness scale scores.

\section{REFERENCES}

1. Cystic Fibrosis Foundation. Patient registry annual data report 2012. http://www.cff.org/UploadedFiles/research/ClinicalResearch/ PatientRegistryReport/2012-CFF-Patient-Registry.pdf. Accessed April 17, 2015.

2. Boyle MP. Adult cystic fibrosis. JAMA 2007;298(15):1787-1793.

3. Abbott J, Hart A, Havermans T, Matossian A, Goldbeck L, Barreto $\mathrm{C}$, et al. Measuring health-related quality of life in clinical trials in cystic fibrosis. J Cyst Fibros 2011;10(Suppl 2):S82-S85.

4. Elkins MR, Robinson M, Rose BR, Harbour C, Moriarty CP, Marks $\mathrm{GB}$, et al. A controlled trial of long-term inhaled hypertonic saline in patients with cystic fibrosis. N Engl J Med 2006;354(3):229-240.

5. McCoy KS, Quittner AL, Oermann CM, Gibson RL, Retsch-Bogart GZ, Montgomery AB. Inhaled aztreonam lysine for chronic airway Pseudomonas aeruginosa in cystic fibrosis. Am J Respir Crit Care Med 2008;178(9):921-928.

6. Abbott J, Hart A. Measuring and reporting quality of life outcomes in clinical trials in cystic fibrosis: a critical review. Health Qual Life Outcomes 2005;3:19.

7. Weiner JR, Toy EL, Sacco P, Duh MS. Costs, quality of life and treatment compliance associated with antibiotic therapies in patients with cystic fibrosis: a review of the literature. Expert Opin Pharmacother 2008;9(5):751-766.

8. Hadjiliadis D. Special considerations for patients with cystic fibrosis undergoing lung transplantation. Chest 2007;131(4):1224-1231.

9. The WHOQOL Group. The World Health Organization quality of life assessment (WHOQOL): development and general psychometric properties. Soc Sci Med 1998;46(12):1569-1585.

10. Skevington SM, Lotfy M, O'Connell KA. The World Health Organization's WHOQOL-BREF quality of life assessment: psychometric properties and results of the international field trial. A report from the WHOQOL group. Qual Life Res 2004;13(2):299-310. 


\section{HRQOL IN CF}

11. Quittner AL, Buu A, Messer MA, Modi AC, Watrous M. Development and validation of the Cystic Fibrosis Questionnaire in the United States: a health-related quality-of-life measure for cystic fibrosis. Chest 2005;128(4):2347-2354.

12. Gee L, Abbott J, Conway SP, Etherington C, Webb AK. Development of a disease specific health related quality of life measure for adults and adolescents with cystic fibrosis. Thorax 2000;55(11):946954.

13. Gee L, Abbott J, Hart A, Conway SP, Etherington C, Webb AK. Associations between clinical variables and quality of life in adults with cystic fibrosis. J Cyst Fibros 2005;4(1):59-66.

14. Riekert KA, Bartlett SJ, Boyle MP, Krishnan JA, Rand CS. The association between depression, lung function, and health-related quality of life among adults with cystic fibrosis. Chest 2007;132(1): 231-237.

15. Simon SL, Duncan CL, Horky SC, Nick TG, Castro MM, Riekert KA. Body satisfaction, nutritional adherence, and quality of life in youth with cystic fibrosis. Pediatr Pulmonol 2011;46(11):1085-1092.

16. Young AC, Wilson JW, Kotsimbos TC, Naughton MT. The impact of nocturnal oxygen desaturation on quality of life in cystic fibrosis. J Cyst Fibros 2011;10(2):100-106.

17. Smyth AR, Bell SC, Bojcin S, Bryon M, Duff A, Flume P, et al. European Cystic Fibrosis Society Standards of Care: Best Practice guidelines. J Cyst Fibros 2014;13(Suppl 1):S23-S42.

18. Fleck MP, Chachamovich E, Trentini C. Development and validation of the Portuguese version of the WHOQOL-OLD module. Rev Saude Publica 2006:40(5):785-791.

19. Rozov T, Cunha MT, Nascimento O, Quittner AL, Jardim JR. Linguistic validation of cystic fibrosis quality of life questionnaires. J Pediatr (Rio J) 2006;82(2):151-156.

20. Miller MR, Hankinson J, Brusasco V, Burgos F, Casaburi R, Coates A, et al. Standardisation of spirometry. Eur Respir J 2005;26(2):319338.

21. Pereira CA, Sato T, Rodrigues SC. New reference values for forced spirometry in white adults in Brazil. J Bras Pneumol 2007;33(4): 397-406.

22. Shwachman H, Kulczycki LL. Long-term study of one hundred five patients with cystic fibrosis; studies made over a five- to fourteenyear period. AMA J Dis Child 1958;96(1):6-15.

23. Brasfield D, Hicks G, Soong S, Tiller RE. The chest roentgenogram in cystic fibrosis: a new scoring system. Pediatrics 1979;63(1):24-29.

24. Quiñones MA, Otto CM, Stoddard M, Waggoner A, Zoghbi WA. Recommendations for quantification of Doppler echocardiography: a report from the Doppler Quantification Task Force of the Nomenclature and Standards Committee of the American Society of Echocardiography. J Am Soc Echocardiogr 2002;15(2):167-184.

25. ATS Committee on Proficiency Standards for Clinical Pulmonary Function Laboratories. ATS statement: guidelines for the six-minute walk test. Am J Respir Crit Care Med 2002;166(1):111-117.

26. Soaresa MR, Pereira CA. Six-minute walk test: reference values for healthy adults in Brazil. J Bras Pneumol 2011;37(5):576-583.

27. Buysse DJ, Reynolds CF 3rd, Monk TH, Berman SR, Kupfer DJ. The Pittsburgh Sleep Quality Index: a new instrument for psychiatric practice and research. Psychiatry Res 1989;28(2):193-213.
28. Johns MW. A new method for measuring daytime sleepiness: the Epworth Sleepiness Scale. Sleep 1991;14(6):540-545.

29. Bertolazi AN, Fagondes SC, Hoff LS, Pedro VD, Menna Barreto SS, Johns MW. Portuguese-language version of the Epworth Sleepiness Scale: validation for use in Brazil. J Bras Pneumol 2009;35(9):877883

30. Bertolazi AN, Fagondes SC, Hoff LS, Dartora EG, Miozzo IC, de Barba ME, Barreto SS. Validation of the Brazilian Portuguese version of the Pittsburgh Sleep Quality Index. Sleep Med 2011;12(1): 70-75.

31. American Academy of Sleep Medicine. The AASM manual for the scoring of sleep and associated events: rules, terminology and technical specifications. Darien, IL: AASM; 2012.

32. American Academy of Sleep Medicine. International classification of sleep disorders: diagnostic and coding manual, 2nd edition. Darien, IL: AASM; 2005.

33. Goldbeck L, Schmitz TG, Henrich G, Herschbach P. Questions on life satisfaction for adolescents and adults with cystic fibrosis: development of a disease-specific questionnaire. Chest 2003;123(1): $42-48$

34. Gee L, Abbott J, Conway SP, Etherington C, Webb AK. Validation of the SF-36 for the assessment of quality of life in adolescents and adults with cystic fibrosis. J Cyst Fibros 2002;1(3):137-145.

35. Spieth LE, Harris CV. Assessment of health-related quality of life in children and adolescents: an integrative review. J Pediatr Psychol 1996;21(2):175-193

36. Bouka A, Tiede H, Liebich L, Dumitrascu R, Hecker C, Reichenberger F, et al. Quality of life in clinically stable adult cystic fibrosis out-patients: associations with daytime sleepiness and sleep quality. Respir Med 2012;106(9):1244-1249.

37. Yohannes AM, Willgoss TG, Fatoye FA, Dip MD, Webb K. Relationship between anxiety, depression, and quality of life in adult patients with cystic fibrosis. Respir Care 2012;57(4):550-556.

38. Abbott J, Hurley MA, Morton AM, Conway SP. Longitudinal association between lung function and health-related quality of life in cystic fibrosis. Thorax 2013;68(2):149-154.

39. Kelemen L, Lee AL, Button BM, Presnell S, Wilson JW, Holland AE. Pain impacts on quality of life and interferes with treatment in adults with cystic fibrosis. Physiother Res Int 2012;17(3):132-141.

40. Shoff SM, Tluczek A, Laxova A, Farrell PM, Lai HJ. Nutritional status is associated with health-related quality of life in children with cystic fibrosis aged 9-19 years. J Cyst Fibros 2013;12(6):746-753.

41. Dill EJ, Dawson R, Sellers DE, Robinson WM, Sawicki GS. Longitudinal trends in health-related quality of life in adults with cystic fibrosis. Chest 2013;144(3):981-989.

42. Bradley J, McAlister O, Elborn S. Pulmonary function, inflammation, exercise capacity and quality of life in cystic fibrosis. Eur Respir J 2001;17(4):712-715.

43. Singh SJ, Sodergren SC, Hyland ME, Williams J, Morgan MD. A comparison of three disease-specific and two generic health-status measures to evaluate the outcome of pulmonary rehabilitation in COPD. Respir Med 2001;95(1):71-77.

44. Enright PL. The six-minute walk test. Respir Care 2003;48(8):783785 